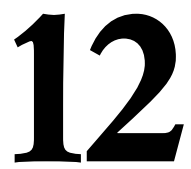

\title{
LA INCIDENCIA DEL SEXO, DEL NIVEL DE COMPETENCIA EN INGLÉS Y DEL GRADO DE MOTIVACIÓN EN PERCEPCIONES SOBRE APRENDIZAJE A TRAVÉS DE UNA APLICACIÓN EN LÍNEA
}

\section{(THE EFFECTS OF SEX, LEVEL OF COMPETENCE IN ENGLISH AND GRADE OF MOTIVATION ON PERCEPTIONS OF LEARNING THROUGH A WEB-BASED PROGRAM)}

Elvira Barrios Espinosa

Universidad de Málaga

DOI: $10.5944 / e d u c X X 1.18 .1 .12333$

\section{Cómo referenciar este artículo/How to reference this article:}

Barrios Espinosa, E. (2015). La incidencia del sexo, del nivel de competencia en inglés y del grado de motivación en percepciones sobre aprendizaje a través de una aplicación en línea. Educación XX1, 18(1), 283-302. doi: 10.5944/educXX1.18.1.12333

Barrios Espinosa, E. (2015). The effects of sex, level of competence in English and grade of motivation on perceptions of learning through a web-based program. Educación XX1, 18(1), 283-302. doi: $10.5944 /$ educXX1.18.1.12333

\section{RESUMEN}

Este artículo explora la incidencia de tres variables, a saber, el sexo, el nivel de competencia en inglés y el grado de motivación, en las percepciones que albergan usuarios de la aplicación en línea Tell Me More acerca de su avance en habilidades comunicativas y competencias lingüísticas mediante dicha aplicación, y acerca de la contribución de distintas actividades que lo conforman a su progresión en inglés. La finalidad que se persigue, en última instancia, es la de identificar características individuales que se relacionan con percepciones más o menos favorables sobre esta aplicación como programa de autoaprendizaje de inglés basado en red. El estudio, en el que participaron 75 docentes de la Universidad de Málaga, empleó el cuestionario como estrategia de recogida de datos, a los que se aplicaron, según los casos, las pruebas estadísticas U de Mann-Whitney y Kruskal-Wallis, y se calcularon tablas de contingencia y el coeficiente de correlación de Kendall. Los hallazgos indican que la variable sexo influye en la percepción de progresión en inglés en tres de las cinco habilidades comunicativas por las que se sondeó a los participantes. Además, se comprueba que, a menor nivel de competencia en inglés, mayor es el grado en que los usuarios consideran que han contribuido las actividades a su aprendizaje; asimismo, de los tres estudiados, el grado de motivación es el factor más 
influyente, tanto en la percepción de avance en habilidades y competencias como en la consideración favorable de la contribución de las distintas actividades al aprendizaje del idioma. Estos hallazgos, junto a la constatación de que, a menor nivel de inglés, mayor es la motivación que genera Tell Me More, lleva a concluir que se trata de una plataforma más recomendable para estudiantes de nivel elemental en inglés que para quienes poseen un nivel igual o superior al nivel B1 según el Marco Común Europeo de Referencia para las Lenguas.

\section{PALABRAS CLAVE}

Aprendizaje de lenguas, uso didáctico del ordenador, autoaprendizaje, tecnología de la educación, características individuales.

\section{ABSTRACT}

This article explores the influence of three variables, namely sex, level of competence in English and grade of motivation, on user perception of their progression in communicative skills and linguistic competences in English by means of the web-based program Tell Me More, and of the contribution of the activities it includes to their progression in English. The ultimate aim is to identify individual factors related to favorable perceptions of this platform as a web-based self-instruction program. Seventy-five teachers at the University of Malaga (Spain) took part in the study, which used a survey as a data-collection method. The Mann-Whitney $U$ and the Kruskal-Wallis tests were applied to the data, and crosstabs and the Kendall correlation coefficient were calculated. Findings indicate that the variable sex influences the perception of progression in English with respect to three out of the five communicative skills investigated. Furthermore, findings point out that the lower the competence level in English, the higher the degree in which users consider that the activities have contributed towards their learning. Also, among the three factors studied, the degree of motivation stands out as the most influential, both for the perception of improvement in communicative skills and linguistic competences and for the favorable consideration of the contribution of the activities to the learning of English. These findings, together with the verification that the lower the competence level in English, the higher the motivation that Tell Me More generates, lead to conclude that it is an English learning platform more suitable for elementary students than for those with a B1 level or above according to the Common European Framework of Reference for Languages.

\section{KEY WORDS}

Language learning, computer use in eduction, self-instruction, educational technology, individual characteristics. 


\section{INTRODUCCIÓN}

Publicaciones recientes atestiguan la creciente demanda y popularidad de aprender idiomas a través de Internet en diferentes modalidades y formatos (sistemas híbridos presenciales y virtuales, métodos virtuales, en contextos de enseñanza presencial o de autoformación que se valen de herramientas y tecnologías web, etc.) (Goodfellow y Lamy, 2009; Meskill y Anthony, 2010; Blake, 2011).

Entre las plataformas diseñadas para el aprendizaje autónomo de idiomas a través del ordenador, Tell Me More figura entre las que gozan de mayor implantación en nuestro entorno. Desarrollado por la compañía francesa Auralog en varios formatos (CD-Rom, soluciones instaladas y aplicación en línea), ofrece un método propio de aprendizaje de idiomas en 5 niveles, los cuales se diseñan conforme a los niveles de competencia A1 a C1 que determina el Marco Común Europeo de Referencia de las Lenguas (MCERL) (Consejo de Europa, 2001).

La aplicación, que incluye una variedad de ejercicios (véase Barrios, 2013, para una descripción detallada de la versión 9 de Tell Me More online), incorpora una sofisticada tecnología de reconocimiento de voz que permite, entre otras funcionalidades, la de visualizar la calidad de la propia producción oral y la de contrastarla con un modelo nativo (Campillos, 2010; Blake, 2011; Delmonte, 2011). Además, son abundantes sus componentes audiovisuales e interactivos (Godwin-Jones, 2010) y se le reconoce su facilidad de uso y la amigabilidad de su interfaz (Lafford, 2004). En el terreno didáctico, aunque varios estudios identifican deficiencias y aspectos de mejora de esta aplicación (Lafford, 2004; Lafford, Lafford y Sykes, 2007; Bidlake, 2009; Nielson, 2011; Barrios, 2013), también existen investigaciones que evidencian que docentes y parte de los usuarios aprecian su utilidad como instrumento de aprendizaje de un idioma y valoran la aplicación de forma positiva (Barrios, 2013; Downey, 2013).

Desde el curso 2009-2010 y con objeto de desarrollar una de las líneas del Plan Estratégico 2009-2012 de la Universidad de Málaga, su Servicio de Formación del Vicerrectorado de Profesorado, Formación y Coordinación oferta formación gratuita de inglés para el personal docente e investigador, la cual comprende cursos presenciales y a distancia (en este último caso mediante el registro en cursos disponibles en Tell Me More online).

Gracias a su labor de coordinación de estos cursos virtuales desde su implantación, la autora ha constatado que el aprendizaje a través de esta aplicación puede generar reacciones e impresiones muy diferenciadas - e incluso encontradas - entre los usuarios. No obstante, la literatura especializada 
en enseñanza y aprendizaje de idiomas asistida por ordenador no ha estudiado hasta la fecha la incidencia de factores individuales tales como los que abordó el estudio que expone este artículo (el sexo, el nivel de competencia y el grado de motivación) en las percepciones sobre aplicaciones de autoaprendizaje del inglés en línea. Estimamos, sin embargo, que la información que puede derivarse de estudios que exploren el impacto de estas variables en las percepciones sobre herramientas y aplicaciones web como medios de aprendizaje autónomo tienen un valor capital para el diseño de material formativo basado en red, para orientar a potenciales usuarios o para tomar decisiones informadas sobre inversiones en materia de capacitación en idiomas.

\section{REVISIÓN DE LA LITERATURA}

En el ámbito educativo general, las diferencias atribuidas al sexo o género ${ }^{1}$ han constituido con frecuencia el foco de atención de iniciativas de investigación; no obstante, no existen hasta la fecha estudios destinados a establecer las diferencias entre hombres y mujeres en percepciones derivadas del uso de aplicaciones para autoaprendizaje en línea de idiomas. Ante esta carencia, recurriremos a informaciones derivadas de investigaciones en torno a la incidencia de esta variable en el aprendizaje basado en red.

De la revisión de la bibliografía sobre este tema se deduce que la evidencia no es concluyente en cuanto al efecto del género en las experiencias de participantes en cursos o actividades de educación a distancia a través de la red. En este sentido, mientras que varios estudios demuestran que hombres y mujeres perciben la experiencia del aprendizaje en línea de forma desigual en cuanto a aspectos tales como uso de las herramientas de comunicación, rendimiento o motivaciones (p. ej., Chyung, 2007; Price, 2006; Rovai y Baker, 2005; Sullivan, 2001), otros, sin embargo, concluyen que los efectos derivados del género son insignificantes (p. ej., Lu, Yu y Liu, 2003; Astleitner y Steinberg, 2005; Yukselturk y Bulut, 2007, 2009). La revisión de la literatura realizada por Astleiner y Steinberg (2005), la cual incluye un metaanálisis de 14 estudios empíricos (fueron excluidos para el análisis estudios no empíricos o estudios empíricos que no presentaban datos estadísticos), concluye que los efectos de esta variable no son significativos a los niveles postulados por el modelo, aunque no descarta que tales diferencias existan. Más recientemente, y en el contexto concreto de aprendizaje de un idioma a distancia (aunque la investigación incluía participantes que optaron por una modalidad enteramente en línea y otros que lo hicieron por una modalidad con un componente presencial), Coleman y Furnborough (2010) tampoco encontraron diferencias entre hombres y mujeres en cuanto a resultados de aprendizaje. No obstante, como señalaban Astleiner y Stein- 
berg $(2005$, p. 60) «The evidence does not open the "Black box" to inform which specific characteristics of WBL environments help female and male students earn higher achievements and enhance levels of satisfaction». Es precisamente este terreno - más concretamente, el de la motivación generada por una herramienta en línea específica y el de percepción de mejora en el rendimiento en términos de avance en competencia en inglés gracias a las actividades propuestas por la misma- el que pretende explorar nuestra investigación.

La incidencia de la competencia inicial en el idioma - determinados previamente niveles de la misma claramente diferenciados- en las percepciones sobre aprendizaje o sobre aplicaciones de aprendizaje de idiomas a distancia no ha sido objeto de estudio hasta la fecha por investigación alguna. De hecho, el único estudio en el que se ha empleado, como variable, el conocimiento previo del idioma, realizado por Coleman y Furnborough (2010), al tratarse de una investigación en el contexto de un curso de idioma (español) de nivel principiante a distancia - aunque solo exclusivamente en línea para una parte de los participantes - únicamente pudo constatar que un nivel de partida más elevado (aunque, es preciso insistir, siempre limitado a una competencia bastante elemental) es un buen indicador de éxito y de continuación en el aprendizaje del idioma; además, el estudio halló que quienes iniciaban el curso con algún conocimiento de español tenían mayor probabilidad de encontrase en el grupo en el que se identificó mayor grado de motivación.

Del conjunto de los factores afectivos cuya influencia se relaciona tradicionalmente con el éxito en el aprendizaje de una lengua, incluido el aprendizaje asistido por ordenador, la motivación y las actitudes del alumno representan quizás los factores más determinantes (p. ej. Gardner, 1985, 2000; Dörnyei, 1994, 2001; Warshauer, 1996; Ushida, 2005). No obstante, no existen estudios que establezcan relaciones entre determinadas actitudes o grado de motivación y aprendizaje o percepción de aprendizaje en un contexto de aprendizaje autónomo íntegramente en línea, como es el caso de nuestro estudio. Acudiremos, por este motivo, a conclusiones de estudios en los que el aprendizaje en línea de una lengua represente, al menos, un componente relevante del programa de instrucción o que integre un número significativo de alumnos cuya experiencia de aprendizaje a distancia fuera exclusivamente a través de la red.

El estudio de Ushida (2005), realizado en un contexto en el que se combinaba aprendizaje de francés o español mediante la red con una sesión semanal presencial, identificó un mayor grado de control sobre su aprendizaje en el alumnado que seguía este programa frente al que lo hacía a través de una modalidad convencional de clases presenciales, aunque no se encontró 
una correlación estadísticamente positiva entre actitudes y motivación de los estudiantes, ni entre actitudes y rendimiento en los exámenes finales. Por su parte, Coleman y Furnborough (2010) identificaron correlaciones positivas estadísticamente significativas entre disfrute con el curso y percepción de logro del objetivo previsto, entre la obtención del certificado del curso y disfrute con el curso, y entre la obtención del certificado del curso y una percepción de logro del objetivo previsto.

\section{DISEÑO DE LA INVESTIGACIÓN}

\section{Objetivos e interrogantes de la investigación}

Nuestro estudio pretendía un triple objetivo:

a) Explorar la incidencia de tres variables, a saber, el sexo, el nivel de competencia en inglés y el grado de motivación, en las percepciones de usuarios de la aplicación en línea Tell Me More sobre la medida en que su participación en un curso realizado a través de esta aplicación había contribuido a mejorar sus habilidades comunicativas;

b) Investigar en qué medida esas tres variables inciden en el grado en que los usuarios advierten que distintas actividades que incluye esta aplicación en línea contribuyen al avance de su competencia en inglés;

c) Estudiar la influencia del sexo y el nivel de competencia en inglés en el grado de motivación que suscita la aplicación en línea Tell Me More.

Más concretamente, la investigación se planteó responder a los siguientes interrogantes:

1. ¿Existen diferencias estadísticamente significativas entre hombres y mujeres, entre usuarios de diferentes niveles de competencia y entre usuarios que evidencian diferentes grados de motivación en relación con la medida en que consideran que han mejorado sus habilidades comunicativas de escuchar, hablar, interaccionar oralmente, leer y escribir, y sus competencias lingüísticas (en concreto, la competencia sintáctica o gramatical, la léxica y la fonológica) como consecuencia de la realización de un curso de 6 meses de duración a través de la aplicación Tell Me More?

2. ¿Existen diferencias estadísticamente significativas entre hombres y mujeres, entre usuarios de diferentes niveles de competencia y entre usuarios que evidencian diferentes grados de motivación, con 
respecto a la medida en que consideran que 26 actividades diferentes que incluye dicha aplicación han contribuido a la mejora de su competencia en inglés?

3. ¿Existen diferencias significativas entre hombres y mujeres y entre los usuarios de niveles diferentes de competencia en cuanto al grado de motivación que suscita en ellos la aplicación en línea Tell Me More?

\section{Participantes}

Participaron en el estudio un total de 75 docentes de la Universidad de Málaga (39 mujeres y 36 hombres), lo que supone el 61,98\% del total de 121 que, en los cursos 2009-2010 y 2010-2011, estaban matriculados en un curso de inglés a distancia, de 6 meses de duración, a través de la mencionada aplicación. Este curso formaba parte del Plan de Formación de la UMA para el personal docente e investigador y se ofertaba de manera gratuita.

\section{Estrategia de obtención de datos}

Se empleó la encuesta como principal estrategia de obtención de datos, a través de un cuestionario; aunque el cuestionario original era más extenso, se empleó para los propósitos de este estudio una sección del mismo integrada por 40 ítems cerrados, además de otros dos que solicitaban los datos del sexo y del nivel en el que - de entre los 5 que ofertaba la versión 9 de Tell Me More en línea² - el participante había pasado la mayor parte de los 6 meses que duraba el curso (ver anexo).

De los 40 ítems arriba referidos, los 6 primeros de ellos constituían una escala - la «Escala de motivación hacia la aplicación Tell Me More»para cuya elaboración se contemplaron aspectos que han sido discutidos en la bibliografía en torno a aprendizaje de una segunda lengua y motivación extrínseca derivada de los componentes del curso (Dörnyei, 1994) (en nuestro caso, interés de las actividades incluidas y su potencial para contribuir al aprendizaje del inglés que se necesita, el interés de los temas y situaciones en relación con los cuales se practica el contenido, el ajuste a las preferencias de aprendizaje particulares, las características del programa y su potencial como herramienta de aprendizaje autónomo). A los encuestados se les solicitó que expresaran su grado de acuerdo con las afirmaciones contenidas en cada uno de los ítems a través de una escala Likert de 5 puntos (donde 1: Muy en desacuerdo; 2: En desacuerdo; 3: Indiferente o Indeciso/a; 4: De acuerdo; 5: Muy de acuerdo). 
Para evaluar la consistencia interna de la escala procedimos a calcular el coeficiente $\alpha$ de Cronbach (1951), el cual se considera el más adecuado para determinar la fiabilidad de un instrumento cuya puntuación final resulta de un procedimiento sumativo o de adición de puntos (Nunnally, 1978), tal como ocurre en nuestro estudio; el coeficiente $\alpha$ de Cronbach para esta escala de motivación es de 0,959 , lo que nos confirma una muy alta fiabilidad del instrumento.

Otros 8 ítems pretendían recoger la percepción de los encuestados en torno al grado en que consideraban que había avanzado su competencia en inglés a raíz de su participación en el curso, con respecto a 5 habilidades comunicativas (escuchar, hablar, interaccionar oralmente, leer y escribir) y 3 competencias lingüísticas (la sintáctica o gramatical, la léxica y la fonológica), a través de una escala de Likert de 5 puntos (donde 1: Nada; 2: Poco; 3: Regular; 4: Bastante; 5: Mucho).

Los 26 ítems restantes pretendían recoger la percepción de los participantes en el estudio en torno a la medida en que otras tantas actividades incluidas en la aplicación Tell Me More en línea habían contribuido a mejorar su competencia en inglés, a través de una escala Likert de las mismas características que la anterior.

Del total de los 121 participantes en el curso en los años anteriormente citados, el cuestionario fue rellenado electrónicamente a través de la red por 44 informantes, mientras que 31 informantes lo cumplimentaron en papel, en sesiones que tuvieron lugar tres días después de finalizar el curso.

\section{Procedimiento de análisis de los datos}

Con el fin de determinar si existen diferencias significativas entre hombres y mujeres en las percepciones sobre los aspectos de la aplicación Tell Me More en los que se centraba el estudio se aplicó la prueba U de Mann-Whitney.

Con respecto a la variable «nivel» determinamos reducir a tres (básico, intermedio y avanzado) los cinco niveles de competencia en los que participantes del estudio habían trabajado en la plataforma Tell Me More; de este modo, el nivel principiante (A1) y elemental (A2) se unificó en la categoría «nivel básico», el nivel intermedio (B1) se mantuvo como «nivel intermedio» mientras que los niveles «avanzado» (B2) y «experto» (C1) se subsumieron bajo la categoría de «avanzado». Para determinar si existen diferencias significativas entre los participantes de estos tres niveles de competencia en inglés en cuanto a sus percepciones sobre los aspectos de la aplicación que investigaba el estudio se aplicó la prueba no paramétrica de Kruskal-Wallis. 
Por su parte, resolvimos determinar el grado de motivación de los participantes hacia la aplicación Tell Me More a partir de los datos derivados de la «Escala de motivación hacia la aplicación Tell Me More», conformada - como se explicó arriba- por 6 ítems del cuestionario. Con objeto de obtener un índice de motivación se procedió a sumar las puntuaciones asignadas a cada uno de los ítems que componían la escala según la fórmula siguiente: «Muy en desacuerdo»: 1 punto; «En desacuerdo»: 2; «Indiferente o Indeciso/a»: 3; «De acuerdo»: 4; «Muy de acuerdo»: 5, de lo que resultó una medida con un rango posible de valores entre 6 y 30; a continuación, se establecieron 3 grados de motivación en función del sumatorio obtenido en la escala, a saber, un grado "bajo» de motivación (entre 6 y 13 puntos), un grado «intermedio» (entre 14 y 22) y un grado «alto» (entre 23 y 30). Para determinar si existen diferencias significativas en las percepciones sobre los aspectos de la aplicación investigados en el estudio entre los participantes que manifiestan grados de motivación diferenciados se aplicó la prueba no paramétrica de Kruskal-Wallis.

Asimismo, se calcularon las tablas de contingencia para identificar dónde estriban las diferencias entre grupos y se calculó el coeficiente de correlación de Kendall para determinar las características de la asociación entre la variable «nivel» (una vez reducidas las 5 categoría iniciales a 3) y grado de motivación.

Los cálculos estadísticos fueron realizados mediante el programa SPSS, versión 19.

\section{HALLAZGOS Y DISCUSIÓN}

\section{Interrogante 1}

A los datos se les se aplicó la prueba de Mann-Whitney para determinar si existían diferencias significativas entre hombres y mujeres en cuanto a sus percepciones en cuanto a la mejora de sus habilidades comunicativas y sus competencias lingüísticas en inglés como consecuencia de la realización de un curso de 6 meses de duración a través de la aplicación Tell Me More; de las 5 habilidades comunicativas (escuchar, hablar, interaccionar oralmente, leer y escribir) y las 3 competencias lingüísticas (la competencia sintáctica o gramatical, la léxica y la fonológica) por las que se interrogó a los participantes, se identificaron diferencias estadísticamente significativas en las percepciones de mejora relativas a 4 de ellas (la de escuchar (o de comprensión oral) $(U=492,500 ; p=0,021)$, la de leer $(U=468,500 ; p=0,010)$, la de escribir $(U=470,500 ; p=0,011)$ y la competencia léxica $(U=394,000$; $\mathrm{p}=0,001)$ ). Las tablas de contingencia ${ }^{3}$ muestran que, con respecto a todas 
las competencias y habilidades en las que se aprecian diferencias significativas, son invariablemente las mujeres quienes estiman haber mejorado en mayor grado que los hombres.

Para determinar si existen diferencias significativas entre los tres grupos de participantes en función de su nivel de competencia en inglés en las percepciones de mejora en las habilidades comunicativas y competencias lingüísticas arriba referidas, procedimos a aplicar a los datos la prueba de Kruskal-Wallis; únicamente en relación con las percepciones sobre progreso en competencia gramatical se obtuvo una diferencia estadísticamente significativa entre los tres grupos $\left(\chi^{2}=10,561 ; p=0,005\right)$. La tabla de contingencia demuestra que es el grupo conformado por el alumnado con un nivel más bajo de competencia (A1 y A2, según el MCERL), el que sostiene haber progresado en mayor medida en esta competencia lingüística.

Finalmente, con objeto de establecer si existen diferencias significativas entre participantes en las percepciones de mejora en las habilidades comunicativas y competencias lingüísticas arriba referidas en función del grado de motivación («bajo», «medio» o «alto») hacia la aplicación Tell Me More, procedimos a aplicar a los datos la prueba de Kruskal-Wallis, de cuyos resultados se concluye que existen diferencias estadísticamente significativas entre los tres grupos en lo referente al conjunto de las habilidades comunicativas y las competencias lingüísticas (escuchar $\left(\chi^{2}=14,298 ; \mathrm{p}=0,001\right)$; hablar $\left(\chi^{2}=22,854 ; \mathrm{p}=0,000\right)$; interaccionar oralmente $\left(\chi^{2}=22,783 ; \mathrm{p}=0,000\right)$; leer $\left(\chi^{2}=29,902 ; \mathrm{p}=0,000\right)$; escribir $\left(\chi^{2}=30,820 ; \mathrm{p}=0,000\right)$; competencia gramatical $\left(\chi^{2}=35,721 ; \mathrm{p}=0,000\right)$; competencia léxica $\left(\chi^{2}=29,513 ; \mathrm{p}=0,000\right)$ y competencia fonológica $\left(\chi^{2}=33,598 ; \mathrm{p}=0,000\right)$.

Las tablas de contingencia demuestran que es el grupo que manifiesta el grado más elevado de motivación el que sostiene haber progresado en mayor medida en estas habilidades comunicativas y competencias lingüísticas a raíz de su participación en el curso de inglés a distancia mediante la aplicación Tell Me More.

\section{Interrogante 2}

La prueba $U$ de Mann-Whitney $(U=500,500 ; p=0,028)$ revela que únicamente con respecto a la actividad denominada «Vocabulario clave» (la cual consiste en un listado de vocabulario esencial para la lección, junto a su traducción al español y la posibilidad de escucharlo pronunciado en inglés) se dan diferencias significativas entre hombres y mujeres en cuanto a la percepción de la contribución que ha supuesto para su aprendizaje de inglés; la tabla de contingencia evidencia que las mujeres sostienen que 
esta actividad ha contribuido a mejorar su competencia en inglés en mayor medida que los hombres.

La prueba de Kruskal-Wallis determinó que existen diferencias estadísticamente significativas entre los participantes con diferentes niveles de competencia en inglés en lo tocante a la percepción de la contribución para su aprendizaje del inglés en 22 de las 26 actividades por las que se les indagó (Tabla 1); no se detectaron diferencias estadísticamente significativas únicamente en las actividades denominadas "Dictado», "Transformar un texto», «Vídeo y cuestionario»y «Fichas culturales».

\begin{tabular}{|l|r|c|}
\hline \multicolumn{1}{|c|}{$\begin{array}{c}\text { Percepción de mejora en inglés } \\
\text { gracias a la actividad-tipo... }\end{array}$} & $\boldsymbol{\chi}^{\mathbf{2}}$ & $\boldsymbol{p}$ \\
\hline Diálogo: descubrimiento & 19,489 & 0,000 \\
\hline Vocabulario clave & 13,109 & 0,001 \\
\hline Pronunciación de palabras & 6,807 & 0,003 \\
\hline Ejercicio de fonética & 7,214 & 0,027 \\
\hline Palabra correcta & 9,542 & 0,008 \\
\hline Asociación de palabras & 13,252 & 0,001 \\
\hline Diálogo: expresión & 7,961 & 0,019 \\
\hline Completar las frases & 7,761 & 0,021 \\
\hline Crucigrama: traducción & 13,364 & 0,001 \\
\hline Crucigrama: reproducción de audio & 16,545 & 0,000 \\
\hline Asociación imagen-palabra con reconocimiento de voz & 13,166 & 0,001 \\
\hline Palabra correcta con reconocimiento de voz & 16,971 & 0,000 \\
\hline Sopa de letras: reproducción de audio & 20,570 & 0,000 \\
\hline Pronunciación de frases & 12,881 & 0,002 \\
\hline Explicaciones gramaticales clave & 16,042 & 0,000 \\
\hline Palabras y funciones & 14,372 & 0,001 \\
\hline Orden de palabras & 15,552 & 0,000 \\
\hline Casillas de palabras & 15,570 & 0,000 \\
\hline Ejercicio de gramática & 8,235 & 0,016 \\
\hline Diálogo: comprensión & 15,692 & 0,000 \\
\hline Redacción & 9,888 & 0,007 \\
\hline Módulos específicos de vídeo (con reportajes de Euronews) & 6,234 & 0,044 \\
\hline & & \\
\hline
\end{tabular}

Tabla 1. Resultados de la de prueba de Kruskal-Wallis que evidencian la existencia de diferencias estadísticamente significativas entre los participantes con diferentes niveles de competencia en inglés en relación con la percepción de la contribución para su aprendizaje del inglés de 22 actividades de Tell Me More 
Las tablas de contingencia, en su conjunto, evidencian lo siguiente:

- En líneas generales, los participantes con un nivel de competencia en la lengua no superior a un A2 (según la aplicación Tell Me More) reconocen haber mejorado su dominio del inglés gracias a las actividades incluidas en esta plataforma en mayor medida de lo que lo hacen los participantes con un nivel de competencia mayor (en concreto, en relación a 21 de las 22 actividades en las que se evidenciaron diferencias estadísticamente significativas entre grupos según nivel de competencia);

- Los participantes de un nivel intermedio (que corresponde a un nivel B1 en la aplicación) son quienes optan de manera más frecuente por la opción intermedia «Regular» para caracterizar el avance en inglés que le han facilitado las distintas actividades del programa; además, son los que perciben, de forma más frecuente, un menor grado de contribución de las distintas actividades a su competencia en inglés (en concreto, en relación con 13 de las 22 actividades);

- Los participantes pertenecientes al nivel más avanzado de competencia en inglés perciben un menor grado de contribución de las distintas actividades a la mejora de su competencia en inglés con respecto a 9 de las 22 actividades en las que se evidenciaron diferencias estadísticamente significativas entre grupos de nivel de competencia; de esas 9, 6 implicaban trabajar con palabras aisladas, en contraposición al uso del idioma en contextos oracionales o textuales. Por el contrario, este grupo manifestó ser el más beneficiado con la actividad de "Módulos específicos de vídeos», que adoptan un formato de reportaje sobre temas de actualidad, los cuales se acompañan de su transcripción y de preguntas sobre su contenido.

La prueba de Kruskal-Wallis determinó que existen diferencias estadísticamente significativas entre los participantes con diferentes grados de motivación hacia la aplicación Tell Me More, en cuanto a la percepción de la contribución para su aprendizaje del inglés de la totalidad de las actividades por las que se les indagó (Tabla 2).

\begin{tabular}{|l|c|c|}
\hline \multicolumn{1}{|c|}{$\begin{array}{c}\text { Percepción de mejora en inglés } \\
\text { gracias a la actividad-tipo... }\end{array}$} & $\boldsymbol{\chi}^{\mathbf{2}}$ & $\boldsymbol{p}$ \\
\hline Diálogo: descubrimiento & 29,482 & 0,000 \\
\hline Vocabulario clave & 34,556 & 0,001 \\
\hline Pronunciación de palabras & 29,314 & 0,000 \\
\hline Ejercicio de fonética & 20,399 & 0,000 \\
\hline
\end{tabular}




\begin{tabular}{|l|c|c|}
\hline \multicolumn{1}{|c|}{$\begin{array}{c}\text { Percepción de mejora en inglés } \\
\text { gracias a la actividad-tipo... }\end{array}$} & $\chi^{\mathbf{2}}$ & $\boldsymbol{p}$ \\
\hline Palabra correcta & 24,355 & 0,000 \\
\hline Asociación de palabras & 29,921 & 0,000 \\
\hline Diálogo: expresión & 39,706 & 0,000 \\
\hline Completar las frases & 30,598 & 0,000 \\
\hline Crucigrama: traducción & 25,131 & 0,000 \\
\hline Crucigrama: reproducción de audio & 30,136 & 0,000 \\
\hline Asociación imagen-palabra con reconocimiento de voz & 36,379 & 0,000 \\
\hline Palabra correcta con reconocimiento de voz & 27,950 & 0,000 \\
\hline Sopa de letras: reproducción de audio & 23,981 & 0,000 \\
\hline Pronunciación de frases & 19,404 & 0,000 \\
\hline Explicaciones gramaticales clave & 28,406 & 0,000 \\
\hline Palabras y funciones & 37,110 & 0,000 \\
\hline Orden de palabras & 29,268 & 0,000 \\
\hline Casillas de palabras & 33,791 & 0,000 \\
\hline Ejercicio de gramática & 25,291 & 0,000 \\
\hline Diálogo: comprensión & 43,007 & 0,000 \\
\hline Dictado & 21,121 & 0,000 \\
\hline Transforma un texto & 20,727 & 0,000 \\
\hline Redacción & 15,581 & 0,000 \\
\hline Vídeo y cuestionario & 22,698 & 0,000 \\
\hline Fichas culturales & 10,246 & 0,006 \\
\hline Módulos específicos de vídeo (con reportajes de Euronews) & 15,942 & 0,044 \\
\hline
\end{tabular}

Tabla 2. Resultados de la prueba de Kruskal-Wallis que evidencian la existencia de diferencias estadísticamente significativas entre los participantes con diferentes grados de motivación con respecto a la percepción de la contribución para su aprendizaje del inglés de las 26 actividades de Tell Me More por las que se les indagó

Las tablas de contingencia, en su conjunto, evidencian que, de forma invariable, a mayor grado de motivación, mayor satisfacción de los participantes con la contribución al aprendizaje del inglés de las actividades que conforman la aplicación Tell Me More.

\section{Interrogante 3}

Con objeto de obtener una medida del grado de motivación que suscita entre los usuarios la aplicación Tell Me More se empleó, como se explica arriba, la «Escala de motivación hacia la aplicación Tell Me More». 
La prueba U de Mann-Whitney (U=530,000; $p=0,149)$ confirma que no hay diferencia estadísticamente significativa entre hombres y mujeres en lo relativo al grado de motivación que genera la aplicación Tell Me More para aprender inglés en línea de manera autónoma.

Por su parte, la prueba de Kruskal-Wallis $\left(\chi^{2}=19,596 ; \mathrm{p}=0,000\right)$ determinó que existen diferencias significativas entre los participantes de distintos niveles de competencia en inglés en relación con la motivación que suscita en ellos la aplicación Tell Me More como herramienta de aprendizaje autónomo en línea. Analizada la tabla de contingencia, se concluye que el grado de motivación con esta aplicación es mayor cuanto menor es el nivel de competencia en inglés de los usuarios. Este extremo se confirmó mediante el cálculo del coeficiente de correlación de Kendall $(\tau=-464)$, que evidencia que existe una correlación significativa negativa moderada entre el grado de motivación de los participantes en el estudio hacia la aplicación Tell Me More y su nivel de competencia en inglés.

\section{CONCLUSIONES}

Según se desprende de los resultados de nuestro estudio, existen diferencias estadísticamente significativas entre hombres y mujeres en percepción de avance en cuatro de las ocho habilidades comunicativas y competencias lingüísticas por las que se cuestionó a los participantes como consecuencia de su participación en un curso de aprendizaje de inglés a distancia a través de la plataforma Tell Me More. Asimismo se evidencia que, con respecto a esas competencias y habilidades, son las mujeres quienes aprecian haber progresado en mayor medida que los hombres. Por consiguiente, esta investigación se suma a otras (p. ej., Chyung, 2007; Price, 2006; Rovai y Baker, 2005; Sullivan, 2001) que identifican percepciones diferenciadas entre hombres y mujeres en cuanto al aprendizaje a través de Internet y representa un estudio pionero en el terreno del aprendizaje de idiomas mediante una aplicación telemática que encuentra tales diferencias.

La incidencia de la variable «nivel de competencia» en inglés se hace particularmente evidente en las perspectivas que sostienen los participantes sobre la contribución a su aprendizaje del inglés de un considerable número de actividades que incluye este programa. En términos generales, los participantes con nivel más bajo de competencia en inglés (nivel «básico») consideran haber conseguido una mayor progresión en su nivel de dominio del inglés que los participantes de nivel intermedio y avanzado. Este hallazgo parece sugerir que Tell Me More, como herramienta de aprendizaje autónomo, parece más adecuada para quienes cuentan con un nivel de partida principiante o elemental que para estudiantes de inglés de niveles superiores. 
Asimismo, usuarios de nivel avanzado perciben que su competencia en inglés se beneficia escasamente de actividades en las que se aprende mediante palabras aisladas - a diferencia de la consideración más favorable que expresan de las actividades en las que el inglés se expone o practica en forma de muestras de lengua más extensas que la palabra o la oración. Este hallazgo apunta a que una mejora del programa para satisfacer a estudiantes de inglés de niveles intermedio y avanzado debería contemplar el texto -frente a la palabra aislada o la oración- como unidad lingüística de enseñanza.

De las tres variables estudiadas en la investigación en cuanto a sus efectos sobre percepciones de aprendizaje de inglés a través de la plataforma Tell Me More, es el grado de motivación el que demuestra producir un impacto mayor. En esencia, a mayor motivación del usuario hacia el programa Tell Me More, mayor sensación de avance en la totalidad de habilidades comunicativas y competencias lingüísticas por las que se encuestó a los participantes, y mayor satisfacción con la contribución del conjunto de las actividades que la conforman al avance de la competencia en inglés.

Este grado de motivación, aunque no se relaciona de manera significativa con el sexo de los informantes, sí que lo hace con el nivel de competencia en inglés, en el sentido de que un menor nivel de competencia en inglés se corresponde con un mayor grado de motivación.

En definitiva, tanto los datos sobre percepción de aprendizaje mediante Tell Me More como los referidos al grado de motivación que genera esta plataforma apuntan a que se trata de un programa para el aprendizaje autónomo del inglés basado en red más indicado y recomendable para estudiantes con un nivel elemental en inglés que para estudiantes con un nivel igual o superior al nivel B1 según el MCERL, y que parece convencer en mayor medida a usuarias que a usuarios como herramienta de aprendizaje capaz de contribuir a la mejora de habilidades comunicativa que no entrañen producción oral.

\section{NOTAS}

1 En este artículo «sexo» y género» se emplean como términos intercambiables.

2 En la documentación sobre la plataforma Tell Me More se asegura que los niveles en los que el programa de formación está configurado se corresponden con los niveles de competencia especificados en el Marco Común Europeo de Referencia para las Lenguas.

3 La lógica limitación de la extensión del artículo impide incluir las tablas de contingencia realizadas. 


\section{REFERENCIAS BIBLIOGRÁFICAS}

Astleitner, H. y Steinberg, R. (2005). Are There Gender Differences in WebBased Learning? An Integrated Model and Related Effect Sizes. AACE Journal, 13(1), 47-63.

Barrios Espinosa, E. (2013). Aprendizaje de inglés mediante Tell Me More. Perspectivas de los profesores universitarios que utilizan la aplicación en línea. Revista de Educación, 362, 506-534.

Bidlake, E. (2009). Learner Experience Using Self-instructed CALL: Methodological and Learner Insights. NovitasROYAL, 3(2), 93-109.

Blake, R. (2011). Current Trends in Online Language Learning. Annual Review of Applied Linguistics, 31, 19-35.

Campillos Llanos, L. (2010). Tecnologías del habla y análisis de la voz. Aplicaciones en la enseñanza de la lengua. Diálogo de la Lengua II, 1-41. Recuperado de: http://www.dialogodelalengua.com/articulo/pdf/2/1_campillos_DL_2010.pdf

Chyung, S. Y. (2007). Age and gender differences in online behavior, selfefficacy and academic performance. Quarterly Review of Distance Education, 8(3), 213-222.

Coleman, J. A. y Furnborough, C. (2010). Learner characteristics and learning outcomes on a distance Spanish course for beginners. System, 38(1), pp. 14-29.

Consejo de Europa (2001). Marco común europeo de referencia para las lenguas: aprendizaje, enseñanza, evaluación. Madrid: Ministerio de Educación, Cultura y Deporte - Instituto Cervantes Editorial Anaya, 2003.
Cronbach, L. J. (1951). Coefficient alpha and the internal structure of tests. Psychometrika, 16, 297-334.

Delmonte, R. (2011). Exploring Speech Technologies for Language Learning, Speech and Language Technologies, InTech. Recuperado de: http://www.intechopen.com/books/ speech-and-language-technologies/ exploring-speech-technologies-forlanguage-learning

Dörnyei, Z. (1994). Motivation and motivating in the language foreign language classroom. The Modern Language Journal, 78(3), 273-284.

Dörnyei, Z. (2001). New themes and approaches in second language motivation research. Annual Review of Applied Linguistics, 21, 43-59.

Downey, K. (2013). Why Did We Buy That? New Customers and Changing Directions in Collection Development. Collection Management, 38(2), 90-103.

Gardner, R. C. (1985). Social Psychology and Second Language Learning: The role of Attitudes and Motivation. Londres: Edward Arnold.

Gardner, R. C. (2000). Correlation, causation, motivation and second language acquisition. Canadian Psychology, 41, 1-24.

Godwin-Jones, R. (2010). Emerging Technologies: New Developments in Web Browsing and Authoring. Language Learning \& Technology, 14(1), 9-15. Recuperado de http://llt.msu.edu/vol14num1/emerging.pdf

Goodfellow, R. y Lamy, M.-N. (Eds.). (2009). Learning cultures in online education. Londres: Continuum Books. 
Lafford, B. A. (2004). Review of Tell Me More Spanish. Language Learning \& Technology, 8(3), 21-34. Recuperado de http://llt.msu.edu/vol8num3/review1/ default.html

Lafford, B., Lafford, P. y Sykes, J. (2007). Entre dicho y hecho ...: An Assessment of the Application of Research from Second Language Acquisition and Related Fields to the Creation of Spanish CALL Materials for Lexical Acquisition. CALICO Journal, 24(3), 497-529. Recuperado de https://calico.org/html/ article_658.pdf

Lasagabaster, D. y Sierra, J. M. (2003). Students» evaluation of CALL programs. Educational Media International, 40(3-4), 293-304.

Levy, M. (1997). Computer-Assisted Language Learning: Context and Conceptualization. Oxford: Clarendon Press.

Lu, J., Yu, C.-S. y Liu, C. (2003). Learning style, learning patterns and learning performance in a WebCT-based MIS course. Information \& Management, 40, 497-507.

Meskill, C. y Anthony, N. (2010). Teaching Languages Online. Bristol, UK: Multilingual Matters.

Nielson, K. B. (2011). Self-study with language learning software in the workplace: What happens? Language Learning \& Technology, 15(3), 110-129. Recuperado de http://ltt.msu.edu/issues/october2011/nielson.pdf

Nunnally. J. C. (1978). Psychometric Theory. Nueva York: McGraw-Hill.

Price, L. (2006). Gender Differences and Similarities in Online Courses: Challenging Stereotypical Views of
Women. Journal of Computer Assisted Learning, 22, 349-359.

Rovai, A. P. y Baker, J. D. (2005). Gender differences in online learning: Sense of community, perceived learning, and interpersonal interactions. The Quarterly Review of Distance Education, 6(1), 31-44.

Sullivan, P. (2001). Gender differences and the online classroom: male and female college students evaluate their experiences. Community College Journal of Research and Practice, 25, 805-818.

Universidad de Málaga (2008). Plan Estratégico 2009-2012. Recuperado de: http://www.infouma.uma.es/planestrategico/

Ushida, E. (2005). The Role of Students» Attitudes and Motivation in Second Language Learning in Online Language Courses. CALICO Journal, 23(1), 49-78. Recuperado de https://calico. org/html/article_131.pdf

Warschauer, M. (1996). Motivational aspects of using computers for writing and Communications. En M. Warschauer (Ed.), Telecollaboration in foreign language learning: Proceedings of the Hawai»i symposium (Technical Report No. 12, 29-46. Honolulu: University of Hawai»i Second Language Teaching and Curriculum Center.

Yukselturk, E. y Bulut, S. (2007). Predictors for Student Success in an Online Course. Educational Technology \& Society, 10(2), 71-83.

Yukselturk, E. y Bulut, S. (2009). Gender Differences in Self-Regulated Online Learning Environment. Educational Technology \& Society, 12(3), 12-22. 


\section{ANEXO: CUESTIONARIO ORIGINAL UTILIZADO EN ESTE ESTUDIO}

Sexo: $\square$ Mujer $\square$ Hombre

¿En cuál de los niveles que ofrece el programa ha trabajado más tiempo?

Principiante (A1)

Elemental (A2)

Intermedio (B1)

Avanzado (B2)

Experto $(\mathrm{C} 1)$

Indique su grado de acuerdo con las afirmaciones que aparecen a continuación según la siguiente escala: 1: Muy en desacuerdo; 2: En desacuerdo; 3: Indiferente o Indeciso/a; 4: De acuerdo; 5: Muy de acuerdo.

\begin{tabular}{|c|c|c|c|c|c|}
\hline & 1 & 2 & 3 & 4 & 5 \\
\hline Los ejercicios/actividades son interesantes. & & & & & \\
\hline $\begin{array}{l}\text { Los ejercicios/actividades son útiles para aprender el inglés } \\
\text { que necesita. }\end{array}$ & & & & & \\
\hline $\begin{array}{l}\text { Los ejercicios/actividades se ajustan a su estilo o preferencias } \\
\text { de aprendizaje. }\end{array}$ & & & & & \\
\hline Los temas y situaciones que presenta son interesantes. & & & & & \\
\hline $\begin{array}{l}\text { Me he sentido motivado/a a trabajar en el programa por las } \\
\text { características que reúne. }\end{array}$ & & & & & \\
\hline $\begin{array}{l}\text { Creo que es un buen programa para avanzar en inglés de ma- } \\
\text { nera autónoma. }\end{array}$ & & & & & \\
\hline
\end{tabular}

Indique en qué grado cree que ha avanzado su nivel de competencia en inglés en los aspectos que se relacionan a continuación según la escala siguiente: 1: Nada; 2: Poco; 3: Regular; 4: Bastante; 5: Mucho.

\begin{tabular}{|l|l|l|l|l|l|}
\cline { 2 - 5 } \multicolumn{1}{c|}{} & 1 & 2 & 3 & 4 & 5 \\
\hline $\begin{array}{l}\text { Speaking (Expresión oral de tipo monólogo, p. ej. para expo- } \\
\text { ner información) }\end{array}$ & & & & & \\
\hline $\begin{array}{l}\text { Oral interaction (Interacción oral de tipo diálogo o conversa- } \\
\text { ción) }\end{array}$ & & & & & \\
\hline Listening (Comprensión oral) & & & & & \\
\hline Writing (Expresión escrita) & & & & & \\
\hline
\end{tabular}




\begin{tabular}{|l|l|l|l|l|l|}
\cline { 2 - 6 } \multicolumn{1}{c|}{} & 1 & 2 & 3 & 4 & 5 \\
\hline Reading (Comprensión escrita) & & & & & \\
\hline Gramática & & & & & \\
\hline Vocabulario & & & & & \\
\hline Pronunciación & & & & & \\
\hline
\end{tabular}

Indique el grado en que considera que los ejercicios o actividades que se relacionan a continuación le han servido para avanzar en su competencia en inglés según la escala siguiente: 1: Nada; 2: Poco; 3: Regular; 4: Bastante; 5: Mucho.

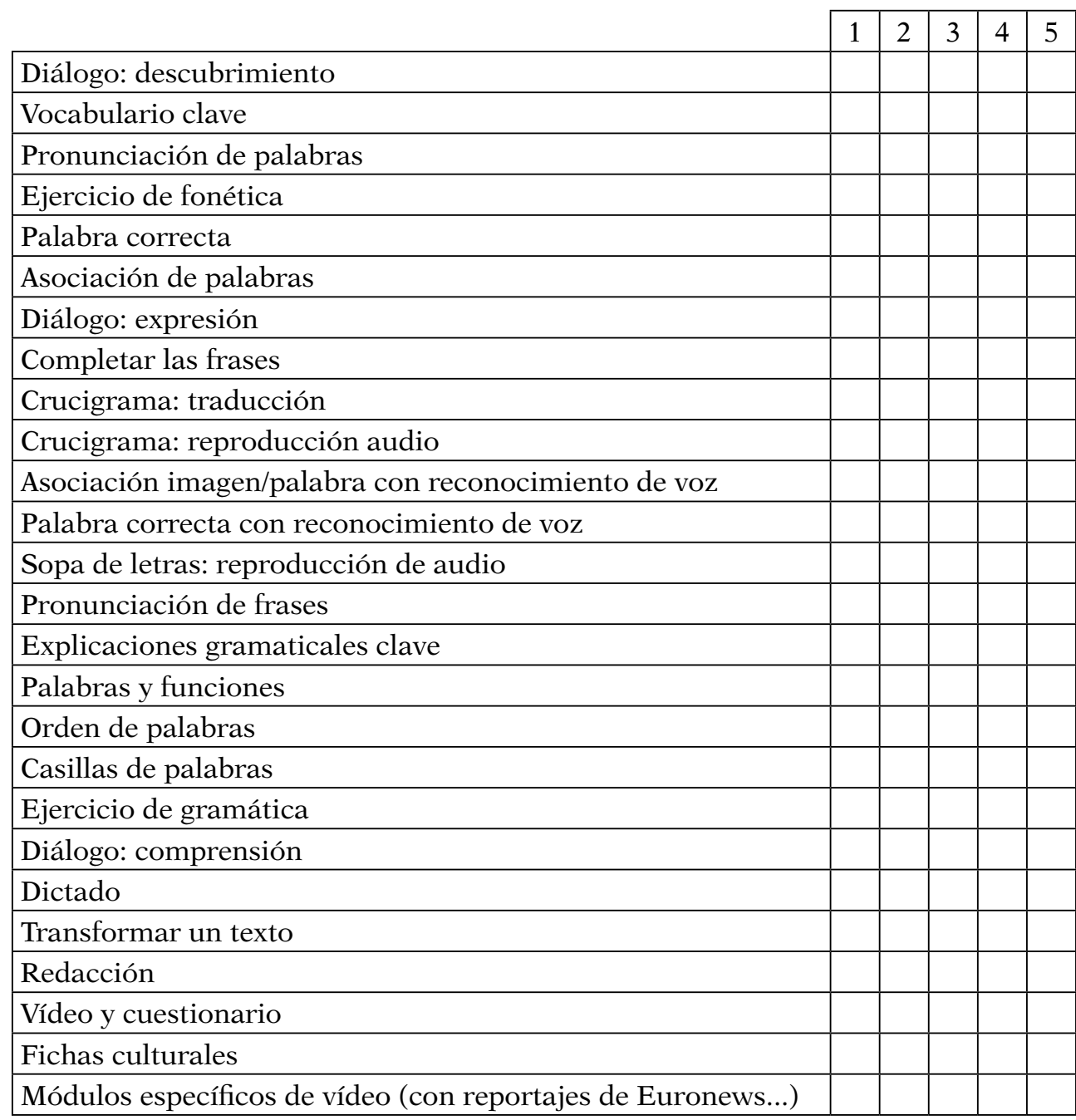




\section{PERFIL DE LA AUTORA}

M. ${ }^{a}$ Elvira Barrios Espinosa, Profesora Titular de Universidad adscrita al Dpto. de Didáctica de la Lengua y la Literatura de la Universidad de Málaga, sus líneas de investigación son las siguientes: concepciones, percepciones y actitudes de alumnos y de profesores en ejercicio y en formación sobre la enseñanza-aprendizaje de una lengua, uso de medios tecnológicos en la enseñanza de lenguas y cuestiones relativas a la implantación de proyectos bilingües en centros educativos.

Dirección de la autora: $\quad$ M. $^{\text {a }}$ Elvira Barrios Espinosa

Dpto. de Didáctica de la Lengua y la Literatura

Facultad de Ciencias de la Educación

Campus de Teatinos

Universidad de Málaga

29071 Málaga

E-mail:m.e.barrios@uma.es

Fecha Recepción del Artículo: 27. Enero. 2012

Fecha Modificación Artículo: 25. Mayo. 2012

Fecha Aceptación del Artículo: 21. Junio. 2012

Fecha Revisión para publicación: 15. Mayo. 2014 